\title{
From axial to road-centre lines: a new representation for space syntax and a new model of route choice for transport network analysis*
}

\author{
Alasdair Turner
}

\begin{abstract}
Axial analysis is one of the fundamental components of space syntax. The space syntax community has suggested that it picks up qualities of configurational relationships between spaces not illuminated by other representations. However, critics have questioned the absolute necessity of axial lines to space syntax, as well as the exact definition of axial lines. Why not another representation? In particular, why not road-centre lines, which are easily available in many countries for use within geographical information systems?

Here we propose that a recently introduced method of analysis, angular segment analysis, can marry axial and road-centre line representations, and in doing so reflect a cognitive model of how route choice decisions may be made. We show that angular segment analysis can be applied generally to road-centre line segments or axial segments, through a simple length-weighted normalisation procedure that makes values between the two maps comparable. We make comparative quantitative assessments for a real urban system, not just investigating angular analysis between axial and road-centre line networks, but also including more intuitive measures based on block-distances between locations.

We show that the new angular segment analysis algorithm produces better correlation with observed vehicular flow than both standard axial analysis and block-distance measures. The results imply that there is no reason why space syntax inspired measures cannot be combined with transportation network analysis representations in order to create a new, cognitively coherent, model of movement in the city.
\end{abstract}

\section{Introduction}

Recently there have been many proposals for new variants of space syntax, which attempt to bring together similar analyses with geographical information science (GISci) (e.g. Jiang and Claramunt, 2002; Batty, 2004; Cutini et al., 2004). These new formulations tend either to automate or to assume procedures to locate key aspects of the skeleton of the street network to form new pointbased representations of urban systems. Within the space syntax community,

* Turner A., 2007. This is a post-print and not the final print version. The definitive, peer-reviewed and edited version of this article is published in Environment and Planning B: Planning and Design 34(3) 539-555, 2007, doi:10.1068/b32067 
there has also been an interest in automation, in order to retrieve an algorithm for the generation of the axial map, a line-based representation of urban systems (e.g. Peponis et al., 1998; Batty and Rana, 2004; Turner et al., 2005).

However, these innovations improve the representation of urban structures rather than the analysis of the representation. In any representation, the cartographer must decide whether or not a certain feature of the environment will be important to a map. The choice she or he makes will affect whatever resulting skeletal network is extracted from it, and thus any representation is ultimately dictated by the cartographer's decision. Therefore, it seems sensible to ask: is there a representation free method of analysing systems? The answer, of course, must be no, since any deviation in the construction of the accompanying graph will, in general, lead to differences in the values of an analytic result. However, it should be possible to devise a system that ensures an analysis with a minimum of interference from the personal preferences of the cartographer.

In this paper, we discuss how a system which is less restricted by the representation may be created for the analysis of topological and skeletal networks alike. We begin with a background to angular segment analysis, and examine why it is an appropriate choice for a backbone for a 'minimum interference' analysis of topological networks. However, angular segment analysis is not just sensible because it can minimise the effects of cartographic differences between representations; there are also strong cognitive grounds for why it should be a good model of pedestrian (and perhaps vehicular) movement. With this in mind, a section on methodology explores what the most appropriate model of movement should be, and how it should be applied.

A section on analysis gives a quantitative of measures for axial maps and road-centre line networks, both for the angular analysis proposed here and blockdistance measures of these networks. As the road-centre line data we use represents vehicular networks, the quantitative analysis is constrained to vehicular road usage, although the methodology could be expanded to pedestrian networks.

It is shown that the new model is a better empirical model of vehicular movement than earlier axial models, with a correlation of up to $R^{2}=0.82$ in an application dataset from the Barnsbury area in London. In addition, it is shown that the angular measures correlate better with movement than methods using shortest block-distance paths between nodes.

These preliminary results are encouraging, and the realisation of the implementation leads to a further conclusion: if space syntax is to be used as a model of movement, then it ought to incorporate ideas from traffic modelling to make a fully coherent model of the built environment. Similarly, if traffic modelling embraces innovations in distance measures, then the accuracy of transportation models could well be considerably improved. By doing so, both communities may arrive at an increased understanding of how society and space interact.

\section{Background}

Angular segment analysis (ASA) has recently seen an upsurge in usage within the space syntax community. In essence, the analysis breaks axial lines into segments, and then records the sum of the angles turned from the starting segment to any other segment within the system (Turner, 2001; Dalton, 2001, 
more details follow in the section on methodology). This angular sum is treated as the 'cost' of a putative journey through the graph, and from it a shortest (that is, least cost) path from one segment to another across the system can be calculated. Most recently, Hillier and Iida (2005) have demonstrated that there is excellent correlation between various ASA measures and movement in four areas of London, including a standard dataset for the Barnsbury area published by Penn and Dalton (1994).

Cognitive scientists have long suggested that angle of turn has much to do with how people perceive the world (Sadalla and Montello, 1989; Montello, 1991; Hochmair and Frank, 2002). Within space syntax, there is also a precedent for angular analysis, stretching back to Penn and Dalton (1994), who show that 'rats' (agents programmed with rules to guide them through the urban maze) which use least-angular strategies for reaching their goals correlate well with patterns of pedestrian movement. More recently, Conroy Dalton (2003) has conducted experiments to show how that people tend to minimise angle towards their destination.

In order apply angular analysis as a graph measure, the author proposed using the measure of 'betweenness' (or 'choice' as it is called in space syntax) in Turner (2000). Betweenness works as follows: for all pairs of possible origin and destination locations, shortest path routes from one to other are constructed. Whenever a node is passed through on a path from origin to destination, its betweenness value is incremented Freeman (1977). Thus, frequently used nodes take high values, while those that fall on fewer paths take low values. Many researchers have noted betweenness seems to be a more intuitive model for movement than the traditional space syntax measure of integration. It has recently been used to assess differences between urban centres (Crucitti et al., 2006) and for angular analysis within space syntax (Hillier and Iida, 2005). However, there is a worry that betweenness is drawn towards densely packed systems of lines, such as found on housing estates, rather than picking out solely highly used routes. Herein, we show both how to avoid this potential problem with betweenness, and how a better correlation can be achieved within the Barnsbury area than that recently presented by Hillier and Iida.

Representational problems associated with axial lines have been highlighted by Ratti (2004). Ratti demonstrates that there may be a change of phase where one axial line suddenly becomes many axial lines due to an apparently minor shift in configuration. ASA does not suffer in this respect, as axial lines split in this way are only at small angle to each other, and the sum weight in both cases is approximately equal. Nevertheless, there are segmented systems where cognitively similar situations result in radically different measurements. The implication is that measures should be chosen according to how they change with a shift in representation.

So what of other representations? Dalton et al. (2003) have already demonstrated the possibility that ASA may be applicable to more than axial maps. Previously, it was thought difficult to apply axial analysis measures to roadcentre lines, due to the fact that road-centre lines may break across junctions, resulting in the segment problem. The segment problem is that axial lines, when broken into segments, have associated higher transfer 'cost' than the straight line, because each step to the next segment incurs a penalty. One response is to make the segments continuous, by joining lines that continue in the current direction to create threads (Thomson, 2003) or continuity lines (Figueiredo 
and Amorim, 2005). Another response is to use angular segment analysis. In ASA, because there is no angular turn to another segment that leads straight on, there is no associated cost, and thus a path that continues in the current direction is by definition continuous across the junction. Dalton et al. apply a variant of angular analysis called fractional analysis Dalton (2001), to show that both geographic road data (in the form of the US national TIGER lines) and axial data have qualitatively similar patterns of analysis values. This should of course come as no surprise to us: the total amount of turn involved on a route between an origin and destination should be approximately the same, regardless of how the topological skeleton is drawn, be it with road-centre lines or axial lines. However, Dalton et al. highlight the relativisation problems between the TIGER data with many lines and the axial data with far fewer lines. In order to fully integrate axial and road-centre lines, we need an analysis free from the effects of numbers of lines, and the answer, I suggest, is to consider the length of segments.

\section{Methodology}

This section starts with a brief review of how angular segment analysis (ASA) works, before turning to the angular measures of mean depth and betweenness. For each measure, I will show how a length-weighted version might be expected to make it representation independent. For completeness, block-distance versions of betweenness and mean depth are also introduced. I then argue that the space syntax concept of 'radius' should also be length-weighted in the same way as the angular and block-distance measures; that is, it should simply be a physical distance. The section ends with a discussion of the implications for syntactic measures in general.

\subsection{Angular segment analysis}

Figure 1 (left) shows a simplified segmented axial map and its associated ' $\mathrm{j}$ graph' ${ }^{1}$ from segment. Firstly, notice that we have simply removed the 'stubs' that overhang in a standard axial map to form a skeletal network of the system; although we do not show it here, stub removal can easily be automated, by using a segmentation routine that cuts off any stub of greater than, for example, $25 \%$ of the overall length of the line. Results when stubs are removed is very similar to the results published herein (Turner, 2005).

To calculate the depth to any location, we calculate the total angular turn from one segment to another segment via the shortest angular route, which we will call $d_{\theta}(x, y)$, where $x$ is the starting segment and $y$ the end segment of the path. We follow Hillier and Iida's (2005) convention, and assign a value in the range of 0 (no turn) to $2\left(180^{\circ}\right.$ turn) for each turn. So, in figure 1 , the depth from segment $P$ to segment $Q$ is 0.5 (a turn of $45^{\circ}$ ) and the depth to segment $R$ is 1.167 (a turn of $45^{\circ}$ followed by a turn of $60^{\circ}$ - note that the direction of turn is immaterial, the turn angle is always positive $\left.{ }^{2}\right)$. When the system is implemented, it becomes obvious that we are not dealing with a straightforward

\footnotetext{
1'Justified graph', that is, the graph as seen from a particular location, in this case, segment $P$.

${ }^{2}$ The figure of 1.167 is a correction to the post-print pointed out by Lucas Figueiredo.
} 

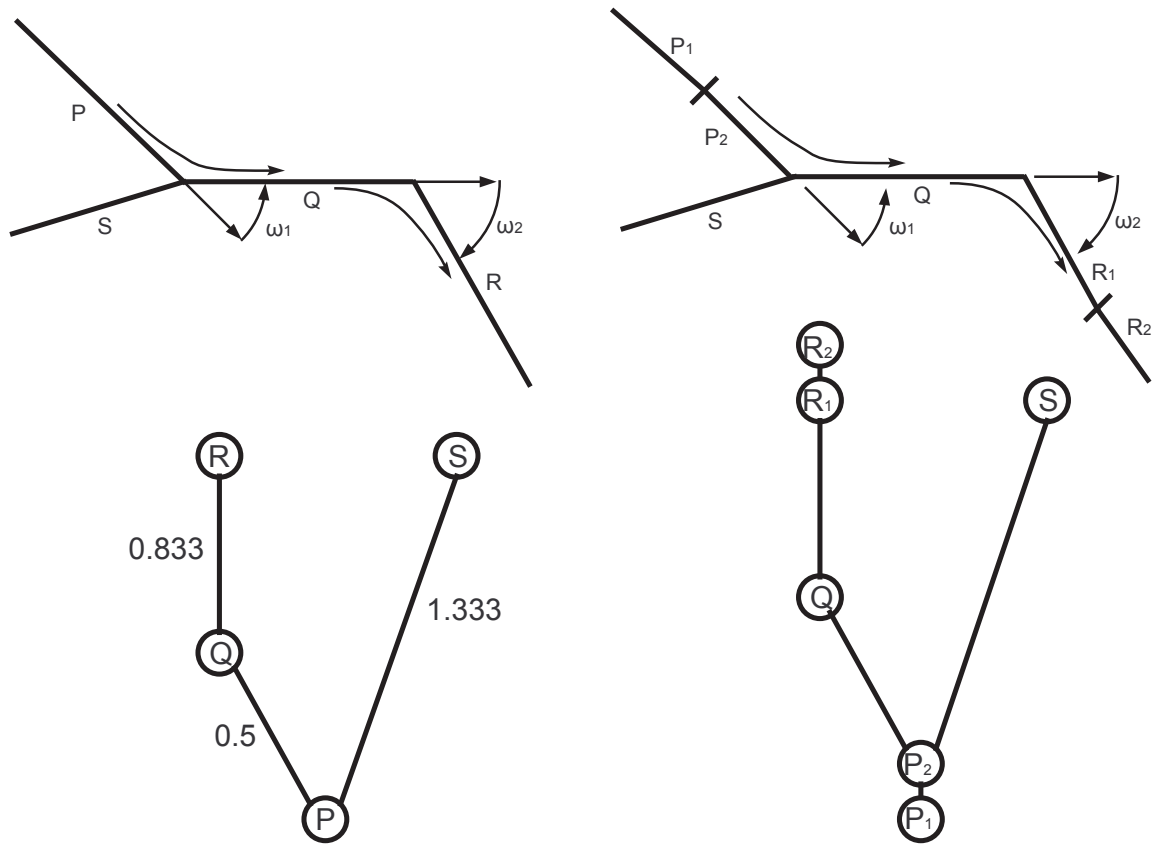

Figure 1: Paths through a network and their associated j-graphs

graph. For example, you cannot move from $P$ to $Q$, then reverse without cost, and move to $S$, claiming that you have turned $45^{\circ}$ to move to $Q$, and then only $15^{\circ}$ to move from $Q$ to $S$. Directionality is important: you must leave from the segment in the same direction as you arrived. Within our implementation ${ }^{3}$ this is handled by incorporating 'back' links and 'forward' links for connections at each end of the segment. If you enter via a 'back' link, then you must leave via a 'forward' link, and vice versa.

\subsection{Angular mean depth}

Space syntax practitioners calculate the mean depth from a location by simply taking the average of all shortest paths, that is:

$$
C_{\theta}(x)=\frac{1}{n} \sum_{i=1}^{n} d_{\theta}(x, i)
$$

In the example in figure 1 , the calculation for location $A$ is as follows: $C_{\theta}(P)=\left(d_{\theta}(P, Q)+d_{\theta}(P, S)+d_{\theta}(Q, R)\right) / 3=(0.5+1.667+1.167) / 3=1.279$. However, what happens when we encounter the situation shown in figure 1 (right)? Here, the cartographer has broken lines $\mathrm{P}$ and $\mathrm{R}$ to more closely follow the situation on the ground, as is usual when drawing road-centre lines. This leads to a mean depth from $A 1$ of $C_{\theta}\left(P_{1}\right)=\left(d_{\theta}\left(P_{1}, P_{2}\right)+d_{\theta}\left(P_{1}, Q\right)+d_{\theta}\left(P_{1}, S\right)+\right.$ $\left.d_{\theta}\left(Q, R_{1}\right)+d_{\theta}\left(Q, R_{2}\right)\right) / 5=(0.033+0.533+1.667+1.2+1.233) / 5=0.933$. So

\footnotetext{
${ }^{3}$ The Depthmap program, which is free for academic use, includes all the analysis algorithms described in this paper.
} 
this small change to representation has made a significant difference to the overall analysis output.

In order to cope with such events, I originally suggested dividing through by the total angular weight within the system rather than the total number of segments Turner (2001). However, the angular weight between one segment and another depends on the direction you are travelling, and so total angular weight varies from segment to segment, which might be seen as problematic in graph terms.

It seems to me to be more intuitive to break the mould of space syntax and think about what we are trying to achieve: an analysis, in this case, of movement patterns. We might expect a longer segment to be associated with a higher percentage of origins and destinations of journeys than a shorter segment (at least within an urban area; the same is not true for a motorway). Thus, it seems sensible to relativise systems by weighting our depth measurements by segment length. We can see how this would work to make the two representations in figure 1 equivalent by thinking about segment $R$. If segment $R$ were $100 \mathrm{~m}$ long, then its contribution to the mean depth is $100 \times 1.167=116.7$. If segment $R_{1}$ is $70 \mathrm{~m}$ long, and segment $R_{2} 30 \mathrm{~m}$ long, then their combined contribution is $70 \times 1.2+30 \times 1.233=121.0$. Obviously, the two are somewhat different due to the extra angles found en route from $P_{1}$ to $R_{2}$, but the overall system mean depth is almost unaffected: if we assume $R$ is actually $100 \mathrm{~m}$ long, then the values of weighted mean depth come out as 1.08 for the left-hand system and 1.09 for the right-hand system. More formally, if $\ell(y)$ is the length of segment $y$, then the weighted mean depth $C_{\theta}^{\ell}$ of segment $x$ in a graph of $n$ segments is defined as follows:

$$
C_{\theta}^{\ell}(x)=\frac{\sum_{i=1}^{n} d(x, i) \ell(i)}{\sum_{i=1}^{n} \ell(i)}
$$

\subsection{Angular betweenness}

Betweenness, or choice as it is called in the space syntax community, is calculated by generating shortest paths between all segments within the system (i.e., the journey with the lowest angular cost for each possible origin and destination pair of segments). We then sum the flow through each segment according to how many journeys are made through each segment, and divide through by the total number of possible journeys ${ }^{4}$ (Freeman, 1977). Normally, a value of ' 1 ' is assigned to every segment passed through on the shortest path from any origin to any destination. Thus, the angular betweenness value for a segment $x$ in a graph of $n$ segments is defined as follows:

$$
B_{\theta}(x)=\frac{\sum_{i=1}^{n} \sum_{j=1}^{n} \sigma(i, x, j)}{(n-1)(n-2)} \text { such that } i \neq x \neq j
$$

\footnotetext{
${ }^{4}$ It has been argued that betweenness is expensive to compute. However, the computational efficiency of a breadth first search to enumerate a shortest path for every OD pair is order $o\left(n^{2}\right)$ where $n$ is the number segments. Choice increases this to order $o\left(n^{2} k\right)$, where $k$ is the average path length, if we introduce a suitable approximation: if two paths have the same angular cost, then choose one or other of the paths at random, rather than split the flow between them. Where many paths exist, as they do in a segment analysis of all possible OD pairs, this stochastic splitting will lead to a good approximation of the choice measure. In testing of large graphs with real number weights such as the ones used here, this approximation made barely noticeable difference (error $<0.1 \%$ ) to the measures.
} 
where $\sigma(i, x, j)=1$ if the shortest path from $i$ to $j$ passes through $x$ and 0 otherwise. However, as we argued before, longer segments are likely to lead to more journeys, simply because more possible origins and destinations may be fitted along them. Thus, we construct a weighted choice measure by multiplying the length of the origin segment by length of the destination segment, and this weight is assigned to each segment on the shortest path. The origin and destination of the path themselves are given half this weight, since we reason that on average one would start and conclude a journey at the middle of each segment. There is no normalisation by the number of possible origins and destinations as the justification for the introduction of the measure is that the total physical length itself that matters to the number of journeys generated, and inter system cannot be conducted otherwise. The weighted choice measure is defined as:

$$
B_{\theta}^{\ell}(x)=\sum_{i=1}^{n} \sum_{j=1}^{n} \sigma^{\ell}(i, x, j) \text { such that } i \neq j
$$

As noted above, the weighted sigma function $\sigma^{\ell}$ used by the $B_{\theta}^{\ell}$ is slightly more complicated the standard one: if the shortest path from $i$ to $j$ passes through $x$, it is simply $\ell(i) \ell(j)$ (length of segment $i$ times length of segment $j$ ); if $x$ is the origin $i$ then $\sigma^{\ell}$ is $\ell(x) \ell(j) / 2$ and if $x$ is the destination $j$, it is $\ell(i) \ell(x) / 2$; otherwise, if $x$ is not on the shortest path between $i$ and $j$, nor the origin or destination of the shortest path from $i$ or $j, \sigma^{\ell}$ is 0 .

\subsection{Block-distance analysis}

It is difficult to incorporate block-distance measures within space syntax, as the representation of the nodes as lines means that an edge between the nodes (a line-line intersection) naturally has no distance component. Hence, most space syntax models have not been tested against block-distance models. However, in order to make any claim about the model being better from a cognitive perspective, we must discount the possibility that people simply follow shortest block-distance paths between origin and destination. Herein, we define the complementary block-distance measures by looking at the midpoint to midpoint distance between segments, as measured along the lines themselves. Thus, if $R_{1}$ is $70 \mathrm{~m}$ long and $R_{2}$ is $30 \mathrm{~m}$ long, and $R_{1}$ is joined to $R_{2}$ as in figure 1 , the blockdistance between them is $15 \mathrm{~m}+35 \mathrm{~m}=50 \mathrm{~m}$, regardless of the angle between them. This results in four analogous measures block distance measures, arrived at simply by substituting the graph distance calculation: block-distance mean depth $\left(C_{l}\right)$, length-weighted block-distance mean depth $\left(C_{l}^{\ell}\right)$, block-distance betweenness $\left(B_{l}\right)$ and length-weighted block-distance betweenness $\left(B_{l}^{\ell}\right)$.

\subsection{Radius}

Radius measures are used within space syntax to avoid edge effect or to observe a local phenomenon. Rather than calculate the graph measure from a segment $x$ to all other segments, the measure is calculated from $x$ to all other segments within a certain number of steps of $x$. Radius calculation suffers similarly to mean depth under different representations, as the number of segments away from a particular location is open to the number of segments that a cartographer uses to represent the feature. As we suggested with mean depth, radius could be 
set up as an angular cost limit, but this raises another common objection with space syntax: very long lines will allow zero transfer cost along their length. This might be applicable to car journeys, but a pedestrian will surely not generally walk the length, for example, of the Edgware Road ${ }^{5}$. In addition, if we are to avoid edge effect (distortions in values due to where we choose to draw the boundary of the graph) it is difficult to constrain a system where certain nodes may connect much more readily into particular corners of the graph. All this leads to the conclusion that we should use a metric radius for our graphs (with the further benefit that 'radius' is simply the standard understanding of the word). This decision has the beneficial effect that our choice measure covers all journeys within a circle of the defined radius and no more. So, if we know the size of our study area, we can define the exact radius we need to take so as to avoid edge effect.

\subsection{Implications for syntactic measures}

Ratti (2004) introduces a paradox to space syntax: that a small configurational change can make a major difference to the representation of the system. The paradox also applies to measures of the representation. Figure 2 shows axial representations of a common feature within the Barnsbury area: urban squares. In figure 2(a) the feature is unclear, and one axial line is draw. In figure 2(b) and $2(\mathrm{c})$, we build up successively wider squares, the single line is gradually split it more lines. There are two problems inherent in this widening. The first is one of relativity. When one line is split into four as in figure $2(\mathrm{~b})$, one gate becomes two gates, and the mean depth increases. However, why should the mean depth increase be commensurate with the flow drop as the occupant takes one path or the other? Indeed, there is also a similar increase in mean depth when we move from figure 2(b) to figure 2(c), but we would surely not expect a similar drop in flow. The second is a problem of flow allocation for the betweenness measure. If the angular turn is higher to the right on figure 2(c) than to the left due to drawing differences, then all the modelled flow will route to the left (there is a similar problem for block-distance measures). In fact, it could be different for the two paths in figure 2(b). However, if there is just a minor deviation, the effect is catastrophic for betweenness: all the flow will take the shorter route. The traffic modelling community realise this problem, and it has led them to introduce probabilistic models of movement, such as logit or probit models (see, e.g., Bell and Iida, 1997). Both choice and mean depth are affected by problems of representation. However, mean depth has a further problem: it cannot provide a direct model of flow. Within space syntax, there is typically a relationship such as $\ln y=m / x+c$, where $y$ is the flow, and $x$ the mean depth. The same is not true of betweenness: because $m$ units of betweenness represent $y$ trips, we are able to drop a parameter and write simply $y=m x$.

\section{Analysis}

As a quantitative test for the representation independent measures, we use data previously gathered for the Barnsbury area in North London and published in

\footnotetext{
${ }^{5}$ In the classic space syntax map of London — shown on the cover of Hillier (1996) Edgware Road, a main artery out of London, is shown as a single line about $5 \mathrm{~km}$ in length.
} 


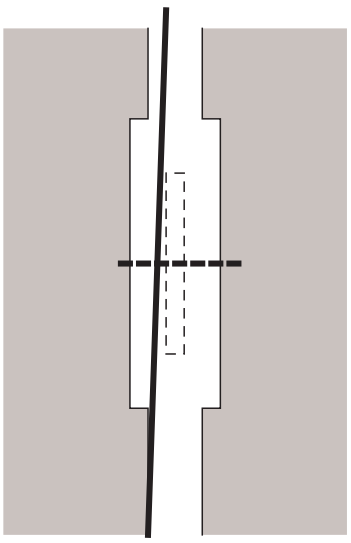

(a)

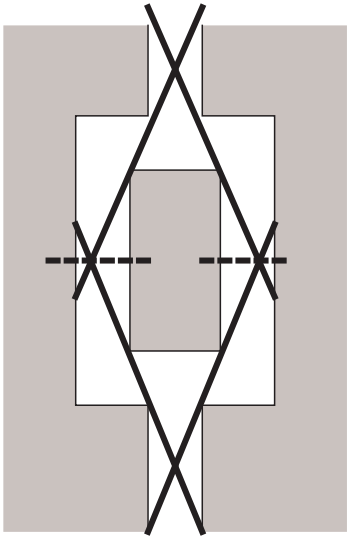

(b)

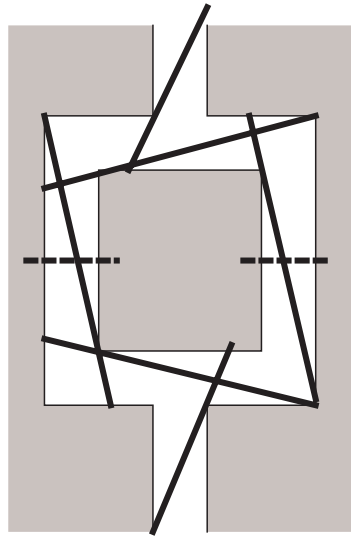

(c)

Figure 2: Axial representations of three different urban squares. Axial lines shown solid; possible gates (locations at which movement is sampled) drawn dashed.

Penn and Dalton (1994). Penn and Dalton took gate counts at nearly every possible inter-junction segment within a roughly $1 \mathrm{~km}^{2}$ area for both vehicular and pedestrian flows. We construct $3 \mathrm{~km} \times 3 \mathrm{~km}$ axial and road-centre line maps around the study area. We first look at the overall pattern of global measures for axial against road-centre line maps, before moving onto a comparison with allday average vehicular movement rates. For measures without radius, we show that axial measures correlate better than road-centre line measures. However, when metric radius is introduced, the road-centre line model can be improved so that it equals the axial model of movement. In both cases the angular measures outperform than the block-distance measures.

We use Ordnance Survey land-line data to construct the road-centre line map, and create an axial map around an original published in Hillier and Hanson (1984). The two maps are shown in figure 3. Note that there are a number of problems with using road-centre line data, which are identified by Dalton et al. (2003): these include topological links that are missed and seemingly arbitrary decisions about whether or not to include road segments. Furthermore, our road-centre line data is taken 10 years after the initial study. We made minor adjustments to the road centre lines to ensure that all links were made where two roads met, and to remove links where barriers prevent traffic flow between segments.

\subsection{Comparison of global measures}

Table 1 shows the average values for measures of the two maps. The roadcentre line map has many more segments than the axial map. As might be expected, the total segment length of the road-centre line map is lower than the axial map, as the axial lines overhang the ends of roads (see back to figure 3 ). Whilst the betweenness values are fairly consistent across representations and methods, the mean depth shows that the systems are very differently configured from the angular point of view. This is perhaps because many more turns are 

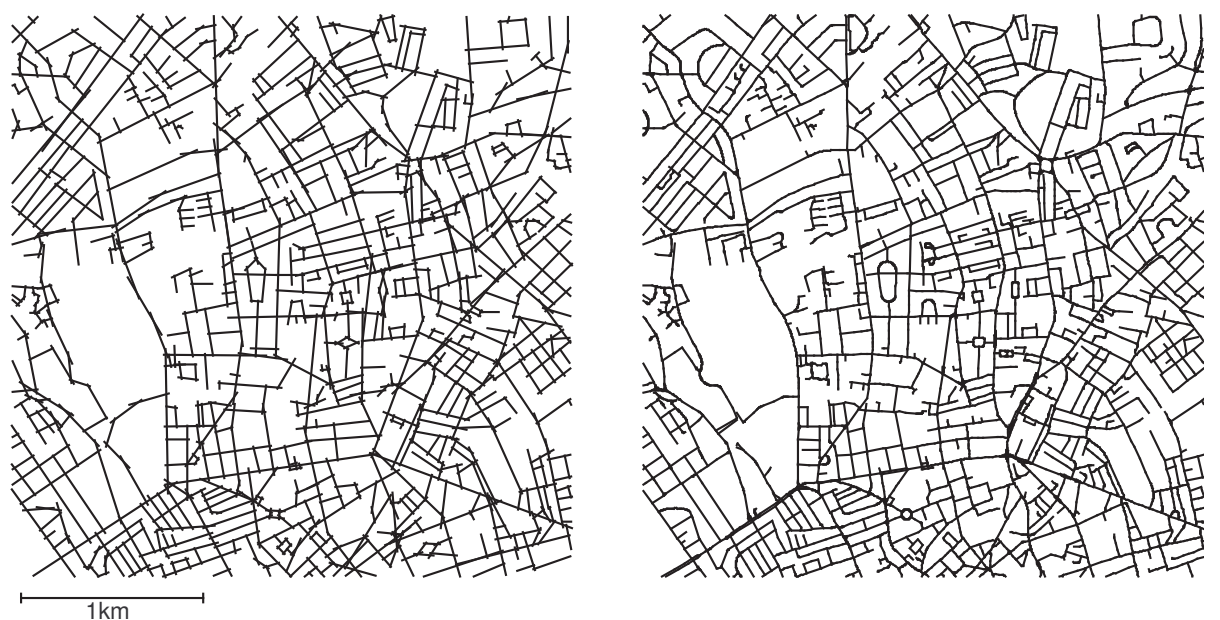

Figure 3: Axial map and road-centre line map of the analysis area. Base map data (C) Crown Copyright/database right 2005. An Ordnance Survey/EDINA supplied service.

modelled in road-centre lines, as meanderings along streets are included in the representation.

\subsection{Cross comparison of global angular measures}

Overall comparison of the values as in the last section is of limited use, as the measures might have similar average values but may be distributed very differently, thus leading to very different movement models. Thus, in order to compare distributions of values, we sample the data at Penn and Dalton's 116 observation gates (figure 4). Note that some gates are on pedestrian paths, so not all can be used for the traffic comparison. We first look at the correlation between the measures themselves, before moving on to the correlation with traffic flow.

Table 1: Average values for measures of axial and road-centre line maps.

\begin{tabular}{lrr} 
Measure & Road-centre line & Axial \\
\hline Angular measures & & \\
$B_{\theta}$ & 0.009 & 0.009 \\
$B_{\theta}^{\ell}$ & $90 \mathrm{~km}^{2}$ & $120 \mathrm{~km}^{2}$ \\
$C_{\theta}$ & 10.77 & 4.79 \\
$C_{\theta}^{\ell}$ & 9.90 & 4.72 \\
Block-distance measures & & \\
$B_{l}$ & 0.010 & 0.009 \\
$B_{l}^{\ell}$ & $108 \mathrm{~km}^{2}$ & $110 \mathrm{~km}^{2}$ \\
$C_{l}$ & $5.41 \times 10^{8}$ & $5.12 \times 10^{8}$ \\
$C_{l}^{\ell}$ & $5.40 \times 10^{8}$ & $5.26 \times 10^{8}$ \\
Total Road Length & $163 \mathrm{~km}^{8}$ & $204 \mathrm{~km}^{2}$ \\
Number of Segments & 20874 & 3933 \\
\hline
\end{tabular}




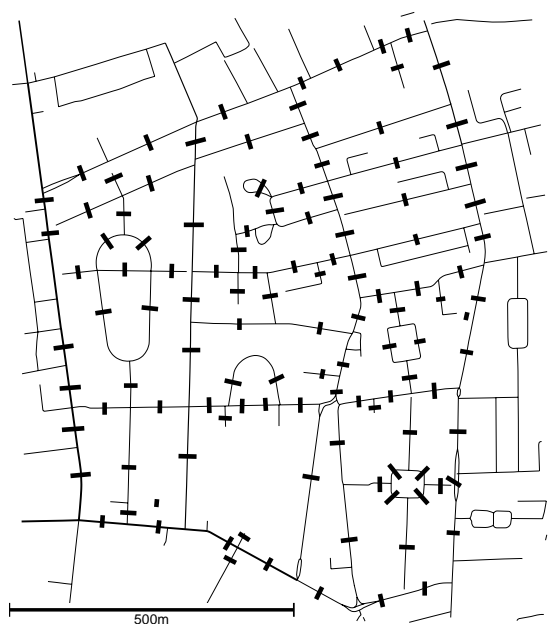

Figure 4: 116 gate locations within the Barnsbury area of North London. Base map data (C) Crown Copyright/database right 2005. An Ordnance Survey/EDINA supplied service.

To correlate the values, the data are normalised by dividing through by the highest observed value of each measure or count (to achieve a value in the range 0 to 1 ), and relativised using a cube root to adjust them to an approximately normal distribution. Table 2 shows a comparison of the global betweenness measures of the axial and road-centre line maps using linear regression, sampled at the 116 gates, whilst table 3 compares the depth measures.

Within each map, there is a strong correspondence between unweighted and weighted measures. However, across types of map, weighting improves the correlation of the measures. Directly comparing angular weighted betweenness on the road-centre line map and axial map gives $R^{2}=0.75$ correlation, and for block-distance weighted betweenness the correlation is also the strongest inter map correlation, with $R^{2}=0.93$. Weighting the mean depth has a similar, but

Table 2: Matrix of $R^{2}$ correlation coefficients for betweenness measures of axial and road-centre line maps.

\begin{tabular}{l|cccc|cccc} 
Analysis & \multicolumn{7}{c|}{ Axial } & \multicolumn{5}{c}{ Road-centre line } \\
& $B_{\theta}$ & $B_{\theta}^{\ell}$ & $B_{l}$ & $B_{l}^{\ell}$ & $B_{\theta}$ & $B_{\theta}^{\ell}$ & $B_{l}$ & $B_{l}^{\ell}$ \\
\hline Axial & \multicolumn{10}{c|}{$\mid$} & & & & \\
$B_{\theta}$ & 1.00 & & & & & & & \\
$B_{\theta}^{\ell}$ & 0.98 & 1.00 & & & & & & \\
$B_{l}$ & 0.67 & 0.66 & 1.00 & & & & & \\
$B_{l}^{\ell}$ & 0.69 & 0.67 & 0.99 & 1.00 & & & & \\
\hline \multicolumn{10}{l}{ Road-centre line } \\
$B_{\theta}$ & 0.72 & 0.76 & 0.56 & 0.59 & 1.00 & & & \\
$B_{\theta}^{\ell}$ & 0.68 & 0.75 & 0.50 & 0.53 & 0.97 & 1.00 & & \\
$B_{l}$ & 0.64 & 0.61 & 0.86 & 0.86 & 0.61 & 0.51 & 1.00 & \\
$B_{l}^{\ell}$ & 0.66 & 0.60 & 0.90 & 0.93 & 0.62 & 0.56 & 0.96 & 1.00 \\
\hline
\end{tabular}


Table 3: Matrix of $R^{2}$ correlation coefficients for mean depth measures of axial and road-centre line maps.

\begin{tabular}{l|cccc|cccc} 
Analysis & \multicolumn{7}{c|}{ Axial } & \multicolumn{5}{c}{ Road-centre line } \\
& $C_{\theta}$ & $C_{\theta}^{\ell}$ & $C_{l}$ & $C_{l}^{\ell}$ & $C_{\theta}$ & $C_{\theta}^{\ell}$ & $C_{l}$ & $C_{l}^{\ell}$ \\
\hline Axial & & & & & & & & \\
$C_{\theta}$ & 1.00 & & & & & & & \\
$C_{\theta}^{\ell}$ & 1.00 & 1.00 & & & & & & \\
$C_{l}$ & 0.27 & 0.26 & 1.00 & & & & & \\
$C_{l}^{\ell}$ & 0.36 & 0.35 & 0.95 & 1.00 & & & & \\
\hline \multicolumn{1}{l}{ Road-centre line } \\
$C_{\theta}$ & 0.65 & 0.67 & 0.16 & 0.27 & 1.00 & & & \\
$C_{\theta}^{\ell}$ & 0.65 & 0.67 & 0.15 & 0.28 & 0.99 & 1.00 & & \\
$C_{l}$ & 0.29 & 0.29 & 0.83 & 0.87 & 0.3 & 0.75 & 1.00 & \\
$C_{l}^{\ell}$ & 0.31 & 0.31 & 0.78 & 0.86 & 0.3 & 0.29 & 1.00 & 1.00 \\
\hline
\end{tabular}

less defined, effect.

Figure 5 shows weighted betweenness measures for the axial and road-centre line maps, while figure 6 shows weighted depth measures for both maps. The figures show that angular mean depth and block-distance mean depth have quite different distributions. This is the fundamental finding of space syntax: that an axial (or this case, angular) centrality measure gives a figure much more similar to betweenness than an expected closeness centrality map. This difference is reflected in the tables: the mean depth for the axial map angular and blockdistance measures do not correlate at all.

Betweenness is much more consistent between maps, but there is a difference between the angular and block distance varieties. The metric measure appears to pick out 'rat-runs' (shortest distance routes through back streets) rather than what appears to be the main road structure as picked out particular by the axial angular betweenness (the thick road the measure picks out at the bottom of the map is the inner London ring road). The road-centre line angular betweenness in turn seems to incorporate a different structure again, influenced perhaps by deviations of angular path length along some of the major routes.

\subsection{Comparison of global measures with traffic flow}

So far, we have not considered the main aim of the analysis, a measure of traffic flow. Table 4 shows the correlation with the average daily traffic flow measured at the gates of both measures. In the table, the regression model 'best-fit' line is simply of the form $y=m x+c$. Clearly, a better model would have actually have traffic strictly proportional to the modelled value (i.e., $c=0$ ). Thus, we also give the value of the intercept $c$ for each model as a fraction of the traffic flow value. Note that $c$ inapplicable to mean depth due to the inverse nature of the relationship.

As might be expected, the block-distance mean depth measure does not correlate at all with movement, as it simply picks out the central area of the analysis zone (see back to figures 5 and 6 ). In addition, in both axial and road centre-line maps the betweenness method is a considerably better correlate with traffic flow than angular mean depth. The road-centre line model appears to 
(a)
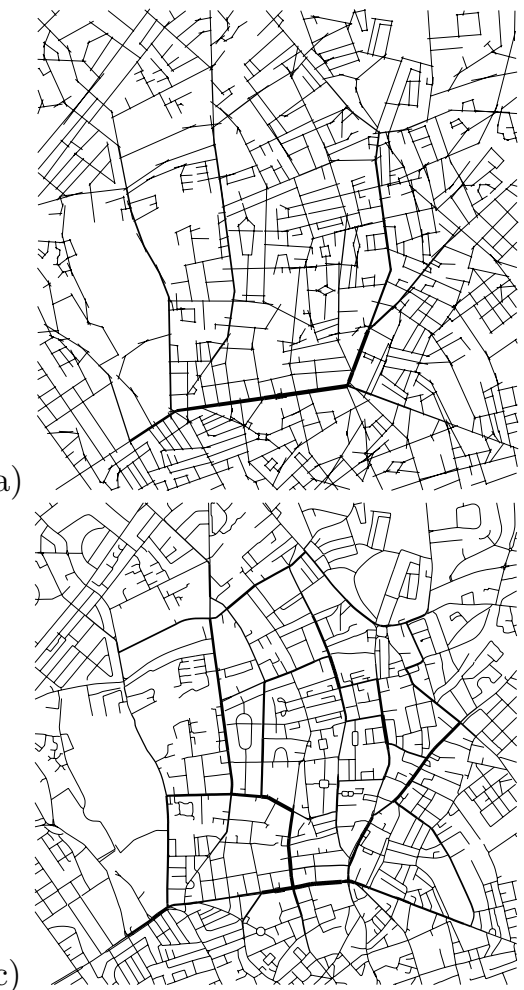

(b)

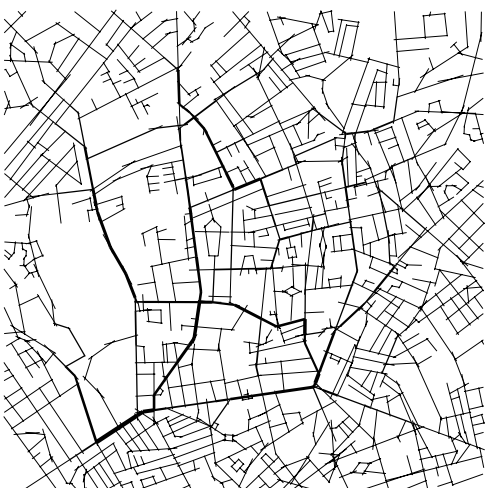

(d)

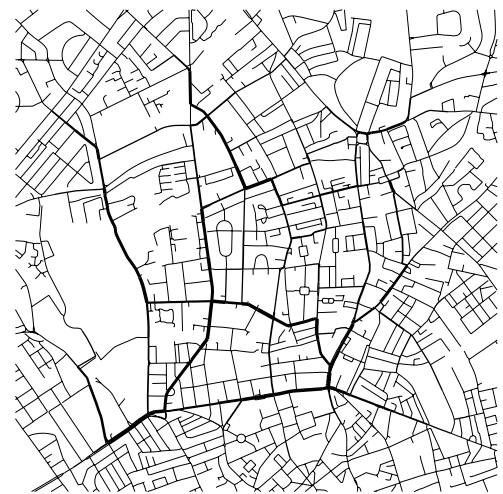

Figure 5: (a) and (b) Weighted betweenness for a $3 \mathrm{~km} \times 3 \mathrm{~km}$ axial system. Thicker lines have higher values. (a) shows angular betweenness; (b) shows block-distance betweenness. (c) and (d) Weighted betweenness for the roadcentre line map of the same area. (c) shows angular betweenness; (d) shows block-distance betweenness. Base map data (c)Crown Copyright/database right 2005. An Ordnance Survey/EDINA supplied service.

Table 4: $R^{2}$ correlation coefficients with observed vehicular flow and intercept for angular and block-distance measures of axial and road-centre line maps.

\begin{tabular}{crrrr} 
Analysis & \multicolumn{2}{c}{$R^{2}$} & $c$ & \multicolumn{2}{c}{$R^{2}$} & $c$ \\
\hline \multicolumn{4}{c}{ Axial } & \multicolumn{2}{c}{ Road-centre line } \\
$B_{\theta}$ & 0.69 & 0.005 & 0.76 & 0.118 \\
$B_{\theta}^{\ell}$ & 0.74 & 0.108 & 0.72 & 0.135 \\
$B_{l}$ & 0.59 & 0.059 & 0.64 & 0.054 \\
$B_{l}^{\ell}$ & 0.61 & 0.029 & 0.66 & 0.011 \\
$C_{\theta}$ & 0.55 & $\mathrm{n} / \mathrm{a}$ & 0.47 & $\mathrm{n} / \mathrm{a}$ \\
$C_{\theta}^{\ell}$ & 0.57 & $\mathrm{n} / \mathrm{a}$ & 0.47 & $\mathrm{n} / \mathrm{a}$ \\
$C_{l}$ & 0.09 & $\mathrm{n} / \mathrm{a}$ & 0.09 & $\mathrm{n} / \mathrm{a}$ \\
$C_{l}^{\ell}$ & 0.12 & $\mathrm{n} / \mathrm{a}$ & 0.09 & $\mathrm{n} / \mathrm{a}$ \\
\hline \multicolumn{5}{c}{}
\end{tabular}


(a)

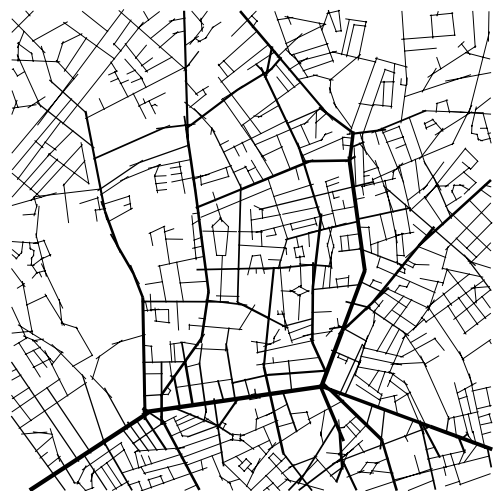

(c)

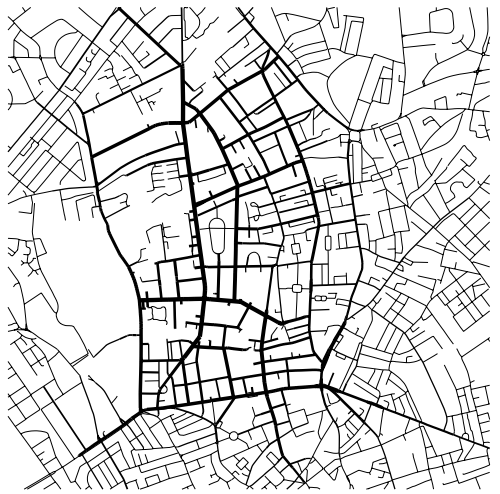

(b)

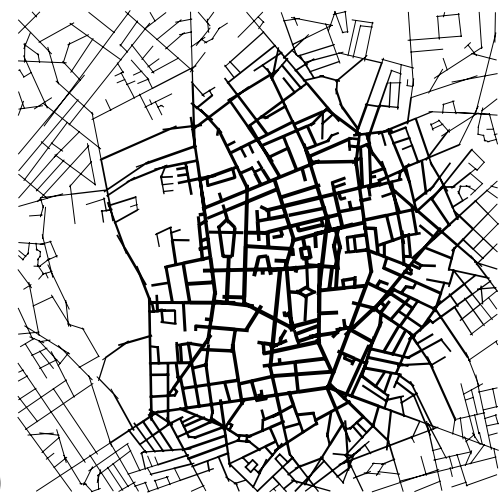

(d)

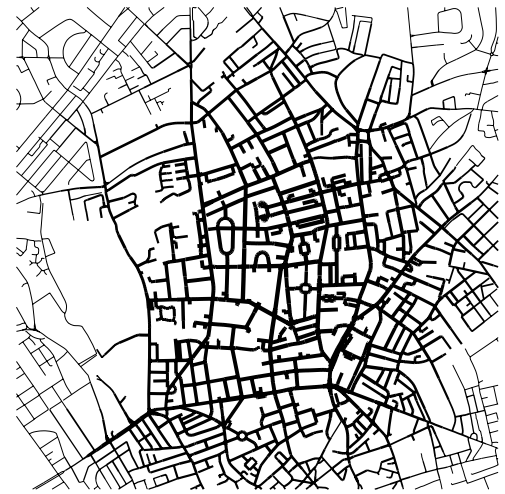

Figure 6: (a) and (b) Weighted mean depth for a $3 \mathrm{~km} \times 3 \mathrm{~km}$ axial system. Thicker lines have lower values (i.e., thicker lines are more central). (a) shows angular mean depth; (b) shows block-distance mean depth. (c) and (d) Weighted mean depth for the road-centre line map of the same area. (c) shows angular mean depth; (d) shows block-distance mean depth. Base map data (C) Crown Copyright/database right 2005. An Ordnance Survey/EDINA supplied service. 

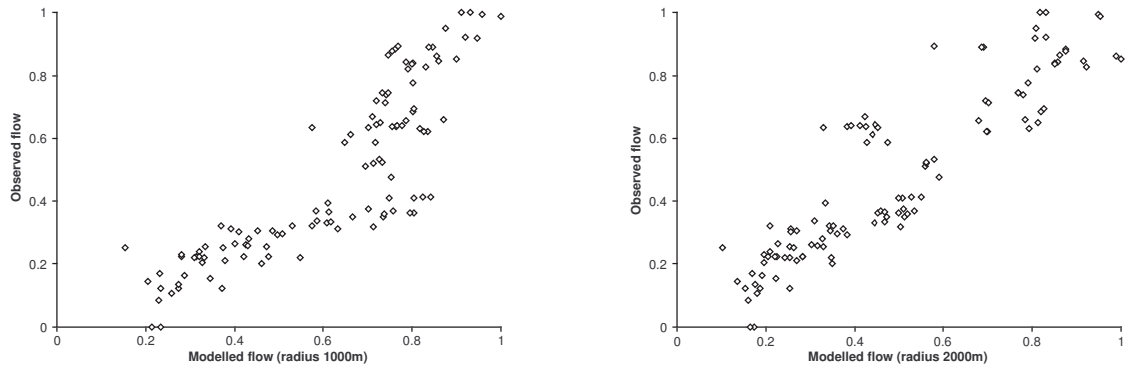

Figure 7: Scatter plots of vehicular movement against weighted choice at radius $1000 \mathrm{~m}$ and radius $2000 \mathrm{~m}$ (adjusted cubic scales as a fraction of maximum observed / modelled flow — see text for details).

perform better both for angular and block-distance betweenness, and angular betweenness seems to correlate better than block-distance betweenness, but we should not expect too much of the figures at this stage: there is surely significant edge effect in the two systems, with only a $1 \mathrm{~km}$ border around the study area. The other noticeable feature is that weighting betweenness for the road-centre line map makes it a slightly worse correlate with traffic flow. At this point, it is tempting to use standard betweenness rather than weighted betweenness for our continued experiments with radius measures. However, the unweighted betweenness measure is a complete artefact of the cartography. In general, curved streets will have more segments than straighter streets, and therefore, within unweighted choice, account for more journeys; that curved streets really will be the origin and destination of more journeys than straight ones of the same length is clearly illogical. Therefore, we continue with the weighted betweenness for the following experiments.

\subsection{Comparison of radius measures}

For radius measures, we will concentrate on the correlation between traffic flow and the modelled weighted choice measure. Radius measures were constructed in the range $1000 \mathrm{~m}$ to $4000 \mathrm{~m}$. Figure 7 shows the general effect of radius for the road-centre line map. At $\mathrm{R} 1000 \mathrm{~m}$ the lower half of the movement is well modelled, whereas above a certain threshold the model breaks down because longer journeys that are supported by the major thoroughfares are not included in the model. As we increase the radius to $2000 \mathrm{~m}$, this effect disappears, and we see the data approach linearity. There are many observations we could make about the groups of outliers observed, but composition of the groups is actually very similar to those found in standard space syntax analysis, and has already seen significant analysis by Penn and Dalton (1994), discussing factors such as the effect of one-way streets.

Figure 8(a) shows how the correlation between observed flow and the weighted betweenness measures vary with radius for both the axial map and the roadcentre line map. The road-centre line map acts as might be expected, with peak correlation at around $2000 \mathrm{~m}$, and then tailing off as the edge effect increases. The shape of the graph is very different from the other measures, which have much flatter graphs, although they still contain peak correlations later. The 

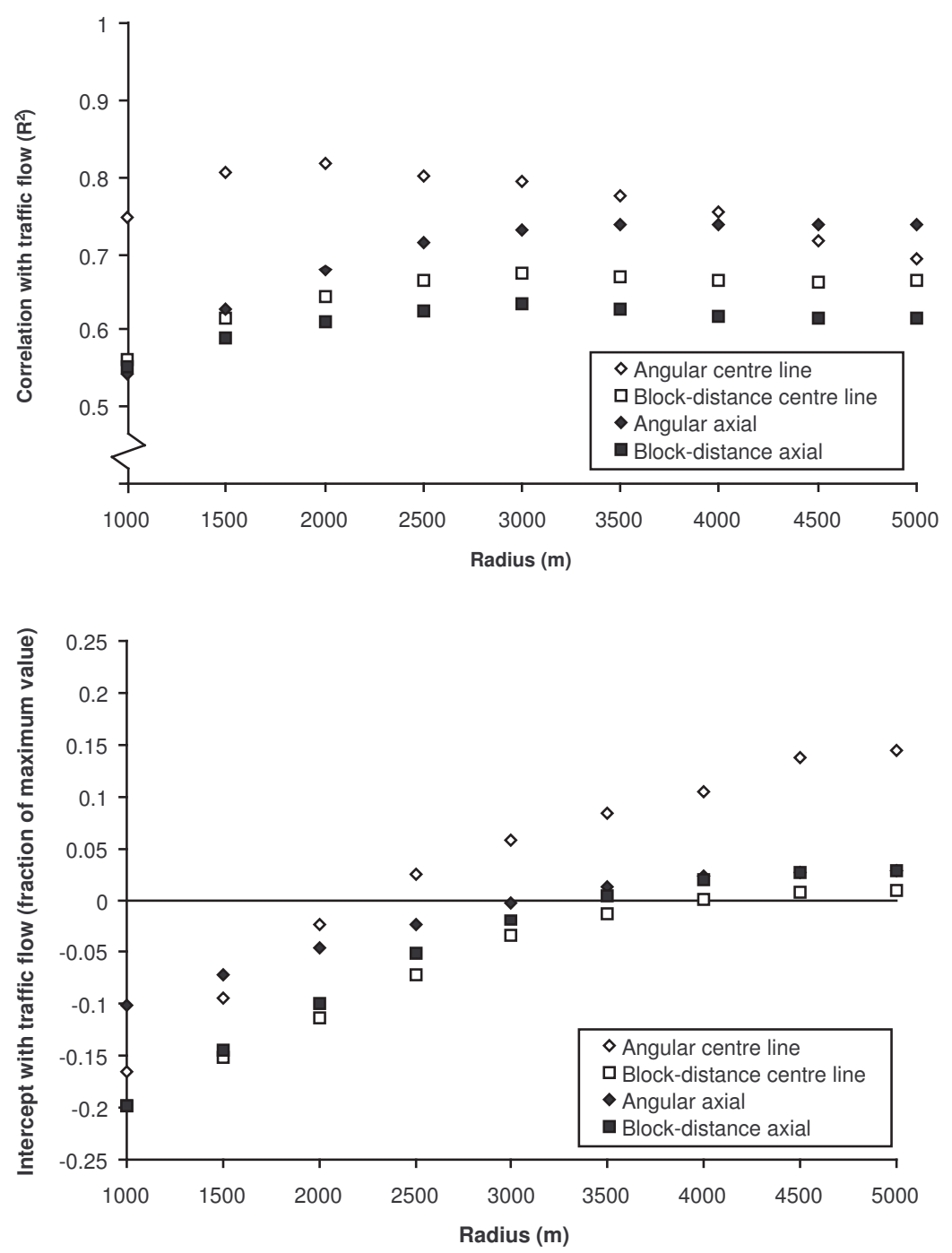

Figure 8: (a) $R^{2}$ correlation coefficients and (b) intercept values for best fit lines of vehicular flow against weighted choice (for both axial and road-centre line maps and block-distance and angular measures). 
maximum correlation for the angular road-centre line model is $R^{2}=0.82$, but $R^{2}=0.67$ for the block-distance model. In both cases the measures are less good for the axial map than the road-centre line map. Figure 8(b) shows the value of the intercept as a fraction of maximum observed flow. As can be seen, in all cases the best-fit line moves up as the radius increases. At about the maximum correlation it moves through the origin itself, demonstrating that the best correlation also corresponds to the best model of vehicular movement.

\section{Conclusion}

In this paper we have shown how road-centre line maps and space syntax axial line maps may be analysed in a comparable fashion by using angular segment analysis (ASA). We discussed the implementation of segment length-weighted versions of centrality (or mean depth) and betweenness (or choice) in order to make the measures of the two representations equivalent. We have argued that betweenness should form a better model of movement than mean depth due to the fact that there is an underlying explanatory model, and that the model is directly proportional to the observed value. We then showed that this is true experimentally for an analysis of the Barnsbury area of North London. We applied the measures to two $3 \mathrm{~km} \times 3 \mathrm{~km}$ maps of the region: one segmented axial map, and one road-centre line map. We found that weighting values made only a small difference to the comparison of the maps, but that betweenness and in particular angular betweenness was a better correlate with observed traffic movement. When the weighted betweenness measures were limited by a metric radius, angular measures of road-centre line yielded a correlation $R^{2}=0.82$ with vehicular flow, whilst the block-distance measures peaked at $R^{2}=0.67$. Whilst both correlate well, the angular measures outperform the block-distance measures.

It seems that the success of a combined road-centre line model analysed using angular weighted betweenness within a metric radius may foster improved space syntactic measures. Those improved measures would still stress the importance of configuration, but would be based on plausible cognitive and physical constraints. For example, we might base a measure on Conroy Dalton's (2003) 'British Library' hypothesis, where it is suggested that people minimise the angle to their destination at each decision point, or revisit Penn and Dalton's pedestrian 'rats' (Penn and Dalton, 1994). In addition, an angular route choice model can be incorporated to more sophisticated models of movement such as found in transportation network analysis, simply by swapping the shortest distance criterion for angle, rather than physical distance. Given that both angle and distance seem to correspond to influence traffic flow, the two methods of route choice might even be combined to reflect genuinely cognitively shortest paths for different levels of knowledge of the system.

\section{Acknowledgements}

This paper is based on 'Could a road-centre line be an axial line in disguise?', originally presented at the 5th International Space Syntax Symposium, TU Delft, Netherlands in 2005. I am grateful for the comments from several re- 
searchers around the world, including in particular Robert Thomson and Sergio Porta, in addition to the anonymous reviewers. The base map data used for the analysis are (C) Crown Copyright/database right 2005. An Ordnance Survey/EDINA supplied service, used with kind permission. More information about the Depthmap software used for the analysis can be found at http: //www.vr.ucl.ac.uk/depthmap

\section{References}

Batty, M, 2004, "A new theory of space syntax", Working Paper 75, Centre for Advanced Spatial Analysis, UCL, London

Batty, M, Rana, S, 2004, "The automatic definition and generation of axial lines and axial maps" Environment and Planning B: Planning and Design 31(4) 615-640

Bell, M G H, Iida, Y, 1997 Transportation Network Analysis (John Wiley and Sons, Chichester)

Conroy Dalton, R, 2003, "The secret is to follow your nose: Route path selection and angularity" Environment and Behavior 35 107-131

Crucitti, P, Latora, V, Porta, S, 2006, "Centrality in networks of urban streets" Chaos 16

Cutini, V, Petri, M, Santucci, A, 2004, "From axial maps to mark point parameter analysis - a GIS implemented method to automate configurational analysis", in Computational Science and its Applications - ICCSA 2004 - Lecture Notes in Computer Science 3044, pp 1107-1116

Dalton, N, 2001, "Fractional configurational analysis and a solution to the Manhattan problem", in Peponis et al. (2001), pp 26.1-26.13

Dalton, N, Peponis, J, Conroy Dalton, R, 2003, "To tame a TIGER one has to know its nature: Extending weighted angular integration analysis to the description of GIS road-center line data for large scale urban analysis", in Hanson (2003), pp 65.1-65.10

Figueiredo, L, Amorim, L, 2005, "Continuity lines in the axial system", in van Nes (2005), pp 163-174

Freeman, L C, 1977, "A set of measures of centrality based on betweenness" Sociometry $4035-41$

Hanson, J (Ed.) 2003, Proceedings of the 4th International Symposium on Space Syntax, UCL, London, UK

Hillier, B, 1996 Space is the Machine (Cambridge University Press, Cambridge, UK)

Hillier, B, Hanson, J, 1984 The Social Logic of Space (Cambridge University Press, Cambridge, UK)

Hillier, B, Iida, S, 2005, "Network effects and psychological effects: A theory of urban movement", in $\operatorname{van}$ Nes (2005), pp 553-564

Hochmair, H, Frank, A U, 2002, "Influence of estimation errors on wayfinding decisions in unknown street networks analyzing the least-angle strategy" Spatial Cognition and Computation 2 283-313 
Jiang, B, Claramunt, C, 2002, "Integration of space syntax into GIS: New perspectives for urban morphology" Transactions in GIS 6(3) 295-309

Montello, D R, 1991, "Spatial orientation and the angularity of urban routes" Environment and Behavior 23(1) 47-69

Penn, A, Dalton, N, 1994, "The architecture of society: Stochastic simulation of urban movement", in Simulating Societies: The Computer Simulation of Social Phenomena Eds N Gilbert, J Doran (UCL Press, London) pp 85-125

Peponis, J, Wineman, J, Bafna, S (Eds) 2001, Proceedings of the 3rd International Symposium on Space Syntax, Georgia Institute of Technology, Atlanta, Georgia

Peponis, J, Wineman, J, Bafna, S, Rashid, M, Kim, S H, 1998, "On the generation of linear representations of spatial configuration" Environment and Planning B: Planning and Design 25 559-576

Ratti, C, 2004, "Space syntax: Some inconsistencies" Environment and Planning B: Planning and Design 31(4) 487-499

Sadalla, E K, Montello, D R, 1989, "Remembering changes in direction" Environment and Behavior 21 346-363

Thomson, R C, 2003, "Bending the axial line: Smoothly continuous road centre-line segments as a basis for road network analysis", in Hanson (2003), pp 50.1-50.10

Turner, A, 2000, "Angular analysis: a method for the quantification of space", Working Paper 23, Centre for Advanced Spatial Analysis, UCL, London

Turner, A, 2001, "Angular analysis", in Peponis et al. (2001), pp 30.1-30.11

Turner, A, 2005, "Could a road-centre line be an axial line in disguise?", in van Nes (2005), pp 145-159

Turner, A, Penn, A, Hillier, B, 2005, "An algorithmic definition of the axial map" Environment and Planning B: Planning and Design 32(3) 425-444

van Nes, A (Ed.) 2005, Proceedings of the 5th International Symposium on Space Syntax, TU Delft, Delft, Netherlands 